\title{
Synthesis, Characterization and Cytotoxicity Evaluation of New Compounds from Oxazol-5(4H)-Ones and 1,2,4-Triazin-6(5H)-Ones Classes
}

\author{
ELENA VALENTINA ROSCA ${ }^{1}$, THEODORA VENERA APOSTOL ${ }^{2 *}$, CONSTANTIN DRAGHICI ${ }^{2}$, OCTAVIAN TUDOREL OLARU $^{3 *}$, \\ LAURA ILEANA SOCEA ${ }^{1}$, LUCIAN ISCRULESCU ${ }^{1}$, GABRIEL SARAMET ${ }^{4}$, FLORICA BARBUCEANU ${ }^{5}$, ELENA MIHAELA PAHONTU ${ }^{6}$, \\ STELIAN BARAITAREANU', STEFANIA FELICIA BARBUCEANU ${ }^{1}$ \\ ${ }^{1}$ Carol Davila University of Medicine and Pharmacy, Faculty of Pharmacy, Organic Chemistry Department, 6 Traian Vuia Str., \\ 020956, Bucharest, Romania \\ ${ }^{2}$ Military Medical Scientific Research Center, 24-28 Gr. Cobalcescu Str., 010195, Bucharest, Romania \\ ${ }^{3}$ Carol Davila University of Medicine and Pharmacy, Faculty of Pharmacy, Pharmaceutical Botany and Cell Biology Department, \\ 6 Traian Vuia Str., 020956, Bucharest, Romania \\ ${ }^{4}$ Carol Davila University of Medicine and Pharmacy, Faculty of Pharmacy, Pharmaceutical Technology and Biopharmacy Department, \\ 6 Traian Vuia Str., 020956, Bucharest, Romania \\ ${ }^{5}$ Institute for Diagnosis and Animal Health, 63 Dr. Staicovici Str., 050557, Bucharest, Romania \\ ${ }^{6}$ Carol Davila University of Medicine and Pharmacy, Faculty of Pharmacy, General and Inorganic Chemistry Department, 6 Traian \\ Vuia Str., 020956, Bucharest, Romania \\ ${ }^{7}$ University of Agronomical Sciences and Veterinary Medicine of Bucharest, Faculty of Veterinary Medicine, Clinical Sciences \\ Department, 105 Splaiul Independentei, 050097, Bucharest, Romania
}

\begin{abstract}
This paper presents the synthesis of new heterocyclic compounds from oxazoles and triazinones classes. Some 2-(4-(4-X-phenylsulfonyl) benzamido)acetic acids were condensed with 4-iodobenzaldehyde in presence of acetic anhydride and sodium acetate, when the new corresponding unsaturated oxazol-5(4H)one were obtained. Through the reaction of these oxazol-5(4H)-ones with phenylhydrazine, in presence of the acetic acid and sodium acetate, the new heterocyclic compounds from 1,2,4-triazin-6(5H)-ones class were obtained. The structures of synthesized compounds have been confirmed by spectral methods (FT-IR, ${ }^{1} \mathrm{H}-\mathrm{NMR},{ }^{13} \mathrm{C}-\mathrm{NMR}$ and MS) and elemental analysis. The cytotoxicity of the compounds was evaluated using the Daphnia magna and Artemia salina bioassays.
\end{abstract}

Keywords: oxazol-5(4H)-one, 1,2,4-triazin-6(5H)-one, cytotoxicity, Daphnia magna, Artemia salina

The heterocyclic compounds with oxazole nucleus arouse the interest of many researchers both in terms of their synthesis and physico-chemical properties, as well as of their biological potential, among them, the oxazolones occupying a special place. Oxazol-5(4H)-ones, also known as azlactones, are considered saturated or unsaturated, depending on the nature of the exocylic bond from the $4^{\text {th }}$ atom of the nucleus. Of these, unsaturated oxazolones present a particular importance both for the organic synthesis, as intermediates in the obtaining of other classes of compounds, as well as for their biological properties $[1,2]$. A large number of derivatives from this class are reported in the literature as having biological activity, such as antitumoral [3,4], antibacterial [5-10], antifungal [5,8,9], anti-inflammatory [11], antioxidant [5,12], tyrosinase inhibitors [13,14].

Among the heterocyclic compounds obtained from unsaturated oxazolones are the triazinones cited in the literature for different biological properties, including antitumoral [15,16], antibacterial [17-19], antifungal [17], antioxidant [18], anti-inflammatory [11], anticonvulsant [20].

In previous research we have synthesized compounds with biologic potential having a diarylsulfone fragment [2128] starting from the literature data which indicate that this fragment is present in the structure of various compounds with biological properties, some of them being also used in therapeutics [24,29-31].

Motivated by these literature data, we initiated the synthesis of a new class of heterocyclic compounds from unsaturated oxazolones and of some triazinones derived from these, which to contain a diarylsulfone fragment in the same molecule, in order to obtain new derivatives with biological potential.

In a first stage, we investigated the cytotoxicity of the new synthesized compounds using Daphnia magna and Artemia salina organisms. Among other screening methods alternative to vertebrate animals, these bioassays are rapid, simple, reproducible and cost-efficient. Various studies demonstrated the usefulness of this method in testing various biological end-points [32-36].

\section{Experimental part \\ Chemistry}

The reactants and solvents used in the synthesis were purchased commercially with high purity and were used without further purification. The melting points of the compounds were determined with a Böetius apparatus and are uncorrected. The elemental analysis was determined using a ECS-40-10 Costech micro-dosimeter. The FT-IR spectra were registered on a Bruker Vertex 70 spectrometer (in KBr pellets). The intensity of IR absorption bands are given as: very strong (vs), strong (s), medium $(\mathrm{m})$ and weak (w). The NMR spectra were recorded on a Varian Gemini 300BB spectrometer at $300 \mathrm{MHz}$ for ${ }^{1} \mathrm{H}-\mathrm{NMR}$ and $75 \mathrm{MHz}$ for ${ }^{13} \mathrm{C}$-NMR. The DMSO-d was used as solvent. The chemical shifts ä are reported in ppm relative to tetramethylsilane (TMS) internal standard and the coupling constants J are expressed in hertz $\mathrm{Hz}$. The abbreviations for multiplicities and descriptors of signals used in the ${ }^{\mathrm{H}} \mathrm{H}$ -

\footnotetext{
*email: theodoraapostol@yahoo.com,octav_olaru2002@yahoo.com
} 
NMR spectra are the following: $s=$ singlet, $d=$ doublet, $d d$ $=$ doublet of doublets, $\mathrm{t}=$ triplet, $\mathrm{m}=$ multiplet, $\mathrm{br}=$ broad signal. For the acquisition of the mass spectra of compounds a APCl (Atmospheric Pressure Chemical Ionization) mass spectrometer was used. For oxazolones, a solution of about $0.5 \mathrm{mg} / \mathrm{mL}$ in chloroform was prepared and then was diluted 10 times with methanol ( $1 \%$ formic acid) for positive ionization, when protonated molecular ions were obtained $[\mathrm{M}+\mathrm{H}]^{+}$. For triazinones a $0.5 \mathrm{mg} / \mathrm{mL}$ solution was diluted 10 times with methanol ( $1 \%$ ammonium carbonate) for negative ionization [M-H]: The solutions of the oxazolones series were passed through the APCl interface with positive ionization and the triazinones solutions were passed through the APCl interface with negative ionization. A solution injection system with a loop mounted on a Reodyne 7725 valve and a Varian Prostar 240 SDM pump operating at a flow rate of $50 \mu \mathrm{L} / \mathrm{min}$ was used to drive the solutions. Protonated molecular ions (in positive ionization) or negative (by removing an acidic proton) were fragmented into the argon collision cell at $1.5 \mathrm{mT}$ Torr. The fragments were scanned with the third quadrupole.

\section{Synthesis and characterization of compounds}

The reaction stages for the synthesis of the title compounds are presented in scheme 1 . For the synthesis of the new heterocycles from oxazolones and triazinones class we started to synthesize the 2-(4-(4-X-phenylsulfonyl) benzamido)acetic acids precursors using a multi-step reaction $[37,38]$. Thus, by the reaction of benzene or other halobenzenes ( $\mathrm{X}=\mathrm{Cl}$ or $\mathrm{Br}$ ) with tosyl chloride, the diarylsulfones 1 were obtained, which by oxidation with chromic anhydride gave the corresponding acids 2 . In the next step, by treating the acids with thionyl chloride, the 4 (4-X-phenylsulfonyl) benzoyl chlorides $\mathbf{3}$ were obtained [24]. The N-acylation of glycine with chlorides 3 conducted to the 2-(4-(4-X-phenylsulfonyl) benzamido)acetic acids 4 $[37,38]$. Further, the condensation of the hippuric acids derivatives $\mathbf{4}$ with 4 -iodobenzaldehyde, in the presence of acetic anhydride and sodium acetate, took place with the obtaining of the new benzylidene-oxazol-5(4H)-ones 5, namely 2-(4-(4-X-phenylsulfonyl)phenyl)-4-(4-iodobenzylidene) oxazol-5(4H)-ones. Finally, 3-(4-(4-X-phenylsulfonyl)phenyl)-5-(4-iodobenzylidene)-2-phenyl-1,2dihydro-1,2,4-triazin-6(5H)-ones 6a-c were obtained by treating of oxazolones 5a-c with phenylhydrazine, in the presence of acetic acid and sodium acetate.

The chemical structures of the new compounds were confirmed by FT-IR, ${ }^{1 H}$-NMR, ${ }^{13}$ C-NMR, MS spectra and elemental analysis.

Synthesis of 2-(4-(4-X-phenylsulfonyl)phenyl)-4-(4iodobenzylidene)oxazol-5(4H)-ones 5a-c

A mixture of $\mathrm{N}$-acylated glycine $4 \mathrm{a}-\mathrm{c}(5 \mathrm{mmol})$, 4iodobenzaldehyde ( $5 \mathrm{mmol}$ ) and sodium acetate $(0.41 \mathrm{~g})$ in acetic anhydride ( $1.8 \mathrm{~mL}$ ) was refluxed for $4 \mathrm{~h}$ with magnetic stirring. To the obtained solution, $2 \mathrm{~mL}$ of ethanol was added and allowed it to stand overnight at cold. The obtained precipitate was filtered off, washed with boiling water and cold ethanol, when a yellow coloured crystalline compound was obtained. The compound was recrystallized from an ethanol-chloroform mixture (1:2, $\mathrm{v}: \mathrm{v})$.

4-(4-Iodobenzylidene)-2-(4-(phenylsulfonyl) phenyl) oxazol-5(4H)-one $5 \mathrm{a}$

m.p. $=244-246^{\circ} \mathrm{C}$; yield $=78 \%$

FT-IR $\left(\mathrm{KBr}, v \mathrm{~cm}^{-1}\right): 3085 \mathrm{~m}, 3062 \mathrm{~m}, 1793 \mathrm{vs}, 1772 \mathrm{~s}$, $1655 \mathrm{~s}, 1580 \mathrm{~s}, 1556 \mathrm{~m}, 1554 \mathrm{~m}, 1479 \mathrm{~m}, 1399 \mathrm{~s}, 1327 \mathrm{vs}$, $1306 \mathrm{~s}, 1290 \mathrm{~s}$, 1160vs, 1098s, 861s, 573s;

${ }^{1} \mathrm{H}-\mathrm{NMR}$ (DMSO-d $\left.{ }^{\prime}, \delta \mathrm{ppm}, J \mathrm{~Hz}\right): 7.41(\mathrm{~s}, 1 \mathrm{H}, \mathrm{H}-18)$, 7.70 (t, 7.3, 1H, H-15), 7.85 (t, 7.3, 2H, H-14, H-16), 7.90 (d,
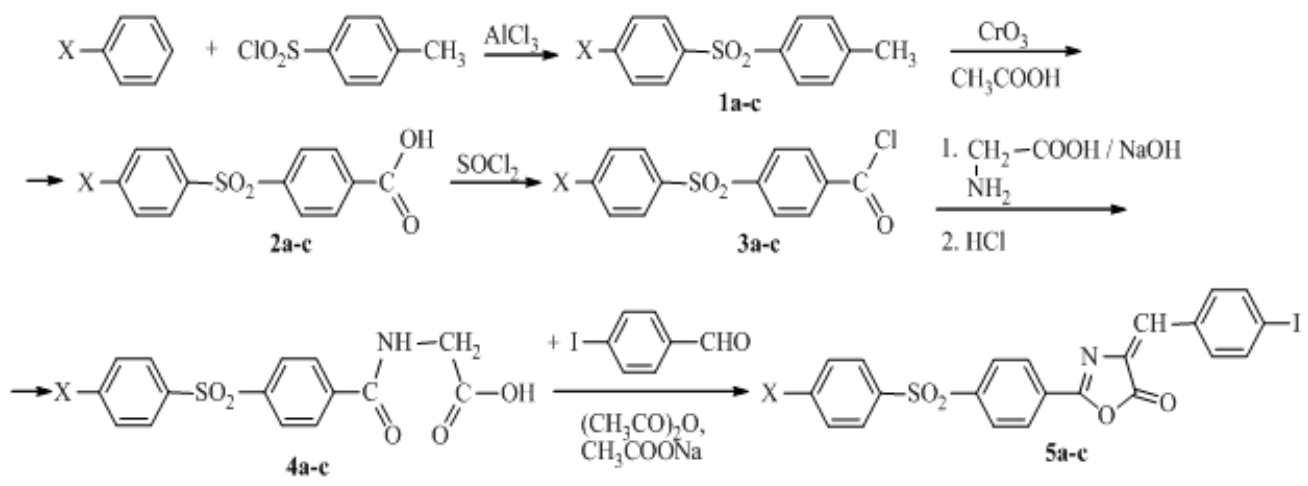

Scheme 1. Synthetic route of compounds 5a-c and 6a-c

$\mathrm{X}: \mathrm{a}=\mathrm{H}, \mathrm{b}=\mathrm{Cl}, \mathrm{c}=\mathrm{Br}$

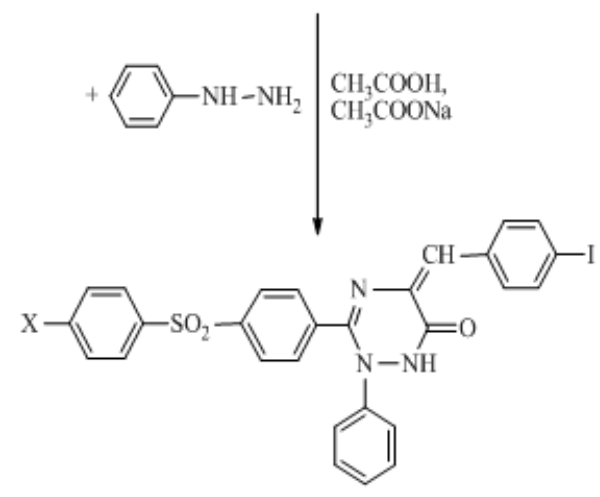

6a-c

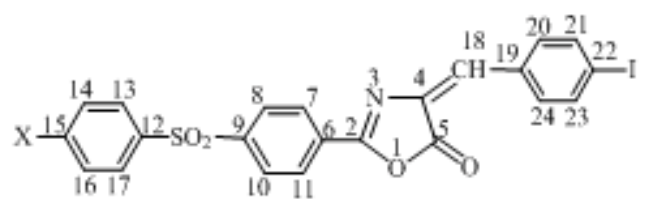

http://www.revistadechimie.ro
Fig. 1. Structure of oxazol-5(4H)-ones 5a-c with atom numbering (for NMR assignments) 
8.6, 2H, H-20, H-24), 7.92 (d, 8.6, 2H, H-21, H-23), 8.00 (br $\mathrm{d}, 7.3,2 \mathrm{H}, \mathrm{H}-13, \mathrm{H}-17), 8.19(\mathrm{~d}, 8.8,2 \mathrm{H}, \mathrm{H}-8, \mathrm{H}-10), 8.31$ (d, 8.8, 2H, H-7, H-11);

${ }^{13} C-N M R$ (DMSO- $d_{6}, \delta$ ppm): 99.81 (C-22), 127.54 (C13, C-17), 128.16 (C-8, C-10), 129.15 (C-7, C-11), 129.61 (C-6), 134.15 (C-15), 129.89 (C-14, C-16), 131.22 (C-18), 132.50 (C-19), 133.40 (C-4), 134.15 (C-20, C-24), 137.95 (C-21, C-23), 140.13 (C-12), 144.76 (C-9), 161.86 (C-2), $166.25(\mathrm{C}-5)$;

Anal. (\%): Calcd. for $\mathrm{C}_{2} \mathrm{H}_{11} I \mathrm{NO} \mathrm{S}(514.97 \mathrm{~g} / \mathrm{mol}): \mathrm{C}$, $51.28 ; \mathrm{H}, 2.74 ; \mathrm{N}, 2.72 ; \mathrm{S}, 6.22$. Found: $\mathrm{C}, 51.31 ; \mathrm{H}, 2.69 ; \mathrm{N}$, 2.80; S, 6.19;

$\mathrm{APCl}, \mathrm{m} / \mathrm{z}(\%): 516[\mathrm{M}+\mathrm{H}]^{+}, 245\left[\mathrm{C}_{6} \mathrm{H}_{5} \mathrm{SO}_{2} \mathrm{C}_{6} \mathrm{H}_{4} \mathrm{CO}\right]^{+}$, $125\left[\mathrm{C}_{6} \mathrm{H}_{5} \mathrm{SO}\right]^{+}$

\section{2-(4-(4-Chlorophenylsulfonyl) phenyl)-4-(4-iodo- benzylidene)oxazol-5(4H)-one $5 \mathrm{~b}$ \\ m.p. $=257-259^{\circ} \mathrm{C}$; yield $=67 \%$}

FT-IR $\left(\mathrm{KBr}, v \mathrm{~cm}^{-1}\right): 3096 \mathrm{~m}, 3075 \mathrm{~m}, 1794 \mathrm{vs}, 1769 \mathrm{~s}$, $1653 \mathrm{~s}, 1577 \mathrm{~s}, 1557 \mathrm{~m}, 1545 \mathrm{~m}, 1474 \mathrm{~m}, 1400 \mathrm{~s}$, $1327 v s, 1291 \mathrm{~s}, 1158 \mathrm{vs}, 1097 \mathrm{~s}, 856 \mathrm{~s}, 768 \mathrm{~s}, 574 \mathrm{~s}$;

${ }^{1} \mathrm{H}-N M R\left(D M S O-d_{6}, \delta \mathrm{ppm}, J \mathrm{~Hz}\right): 7.40(\mathrm{~s}, 1 \mathrm{H}, \mathrm{H}-18)$,

7.75 (d, 8.8, 2H, H-14, H-16), 7.90 ( $\mathrm{m}, 2 \mathrm{H}, \mathrm{H}-2 \mathrm{2}, \mathrm{H}-24), 8.05$ (m, 4H, H-13, H-17, H-21, H-23), 8.20 (d, 8.0, 2H, H-8, H10), $8.30(\mathrm{~d}, 8.0,2 \mathrm{H}, \mathrm{H}-7, \mathrm{H}-11)$;

${ }^{13} \mathrm{C}-\mathrm{NMR}$ (DMSO-d,$\delta \mathrm{ppm}$ ): 99.93 (C-22), 128.35 (C13, C-17), 129.29 (C-8, C-10), 130.15 (C-7, C-11), 129.88 (C-6), 139.03 (C-15), 129.63 (C-14, C-16), 131.37 (C-18), 129.88 (C-19), 133.47 (C-4), 133.90 (C-20,C-24), 138.05 (C-21, C-23), 139.39 (C-12), 144.33 (C-9), 161.90 (C-2), 166.34 (C-5);

Anal. (\%): Calcd. for $\mathrm{C}_{22} \mathrm{H}_{13} \mathrm{ClINO} \mathrm{S}(549.77 \mathrm{~g} / \mathrm{mol})$ : C, 48.06; H, 2.38; N, 2.55; S, 5.83. Found: C, 48.13; H, 2.23; N, 2.62; S, 5.79;

APCl, m/z: $550\left[{ }^{35} \mathrm{Cl} \mathrm{M}+\mathrm{H}\right]^{+}, 552\left[{ }^{37} \mathrm{Cl} \mathrm{M}+\mathrm{H}\right]^{+}, 279$ $\left[{ }^{35} \mathrm{ClC}_{6} \mathrm{H}_{4} \mathrm{SO}_{2} \mathrm{C}_{6} \mathrm{H}_{4} \mathrm{CO}\right]^{+}, 281\left[{ }^{37} \mathrm{ClC}_{6} \mathrm{H}_{4} \mathrm{SO}_{2} \mathrm{C}_{6} \mathrm{H}_{4} \mathrm{CO}\right]^{+}, 159$ $\left[{ }^{35} \mathrm{ClC}_{6} \mathrm{H}_{4} \mathrm{SO}^{2}{ }^{+}, 161\left[{ }^{37} \mathrm{ClC}_{6} \mathrm{H}_{4} \mathrm{SO}\right]^{+}\right.$

2-(4-(4-Bromophenylsulfonyl)phenyl)-4-(4-iodobenzylidene)oxazol-5(4H)-one $5 \mathrm{c}$

m.p. $=281-283^{\circ} \mathrm{C}$; yield $=69 \%$

FT-IR $\left(\mathrm{KBr}, v \mathrm{~cm}^{-1}\right): 3088 \mathrm{~m}, 3043 \mathrm{w}, 1796 \mathrm{vs}, 1772 \mathrm{~s}$, $1654 \mathrm{~s}, 1574 \mathrm{~s}, 1555 \mathrm{~m}, 1544 \mathrm{~m}, 1480 \mathrm{~m}, 1399 \mathrm{~s}, 1328 \mathrm{vs}$, 1307s, 1290s, 1160vs, 1097s, 854s, 615s, 575s;

${ }^{1} \mathrm{H}-\mathrm{NMR}\left(\mathrm{DMSO}-\mathrm{d}_{6}, \delta \mathrm{ppm}, J \mathrm{~Hz}\right): 7.39(\mathrm{~s}, 1 \mathrm{H}, \mathrm{H}-18)$, 7.90-8.04 (m, 6H, H-14, H-16, H-20, H-21, H-23, H-24), 8.05 (d, 8.4, 2H, H-13, H-17), 8.18 (d, 8.4, 2H, H-8, H-10), 8.29 (d, 8.4, 2H, H-7, H-11);

${ }^{13} \mathrm{C}-N M R$ (DMSO-d,$\delta$ ppm): 99.90 (C-22), 128.34 (C13, C-17), 128.79 (C-15), 129.42 (C-7, C-11), 129.61 (C-8, C-10), 132.24 (C-6), 130.27 (C-19), 132.62 (C-18), 132.92 (C-4), 133.42 (C-14, C-16), 134.30 (C-20, C-24), 138.02 (C21, C-23), 139.53 (C-12), 144.26 (C-9), 161.62 (C-2), 166.46 (C-5):

Anal. (\%): Calcd. for $\mathrm{C}_{22} \mathrm{H}_{13}$ BrinO S (594.22 $\left.\mathrm{g} / \mathrm{mol}\right)$ : C, 44.47; H, 2.21; N, 2.36; S, 5.40. Found: C, 44.36; H, 2.30; N, 2.27; S, 5.36;

APCl, m/z: $594\left[{ }^{79} \mathrm{Br} \mathrm{M}+\mathrm{H}\right]^{+}, 596\left[{ }^{81} \mathrm{Br} \mathrm{M}+\mathrm{H}\right]^{+}, 323$ $\left[{ }^{79} \mathrm{BrC}_{6} \mathrm{H}_{5} \mathrm{SO}_{2} \mathrm{C}_{6} \mathrm{HCO}_{4}\right]^{+}, 325\left[{ }^{81} \mathrm{BrC}_{6} \mathrm{H}_{4} \mathrm{SO}_{2} \mathrm{C}_{6} \mathrm{H}_{4} \mathrm{CO}\right]^{+}, 203$ ${ }^{79} \mathrm{BrC}_{6} \mathrm{H}_{4} \mathrm{SO}^{+},{ }^{2} 205\left[{ }^{81} \mathrm{BrC}_{6} \mathrm{H}_{4} \mathrm{SO}\right]^{+}$

Synthesis of 3-(4-(4-X-phenylsulfonyl)phenyl)-5-(4iodobenzylidene)-2-phenyl-1,2-dihydro-1,2,4-triazin-6(5H)ones 6a-c

An equimolar mixture of oxazolone $\mathbf{5}$ and phenylhydrazine $(2 \mathrm{mmol})$ in acetic acid $(5 \mathrm{~mL})$ and in the presence of anhydrous sodium acetate $(0.3 \mathrm{mmol})$ was heated under reflux, with magnetic stirring, for 5 hours.
The reaction mixture was cooled, filtered off and washed with hot water. The obtained product as yellow crystals was recrystalled from ethanol.

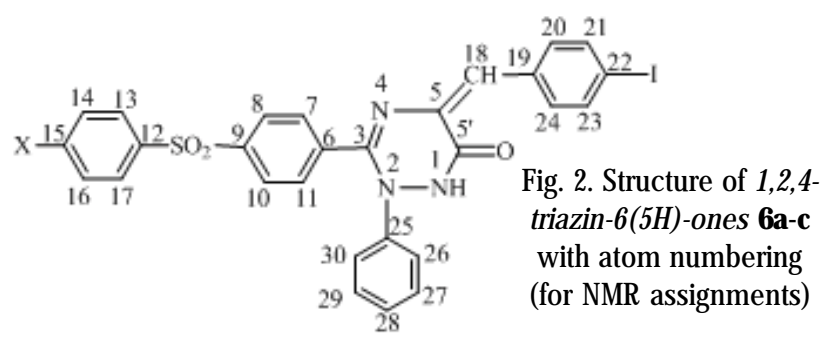

5-(4-lodobenzylidene)-2-phenyl-3-(4-(phenylsulfonyl) phenyl)-1,2-dihydro-1,2,4-triazin-6(5H)-one 6a

m.p. $=272-273{ }^{\circ} \mathrm{C}$; yield $=79.5 \%$

FT-IR $\left(\mathrm{KBr}, v \mathrm{~cm}^{-1}\right): 3281 \mathrm{~m}, 3097 \mathrm{w}, 3065 \mathrm{w}, 3040 \mathrm{w}$, $1722 \mathrm{vs}, 1636 \mathrm{~m}, 1603 \mathrm{~m}, 1578 \mathrm{~m}, 1493 \mathrm{~m}, 1482 \mathrm{~m}, 1405 \mathrm{~m}$, $1321 \mathrm{~m}, 1308 \mathrm{~m}, 1289 \mathrm{~s}, 1154 \mathrm{vs}, 1105 \mathrm{~m}, 819 \mathrm{~m}, 566 \mathrm{~m}$;

${ }^{1} \mathrm{H}-N M R$ (DMSO-d $\mathrm{d}^{\prime} \delta \mathrm{ppm}, \mathrm{J} \mathrm{Hz}$ ): 6.72 (dd, 7.5, 1.1, 2H, $\mathrm{H}-26, \mathrm{H}-30), 6.82(\mathrm{t}, 7.5,1 \mathrm{H}, \mathrm{H}-28), 7.29(\mathrm{~s}, 1 \mathrm{H}, \mathrm{H}-18), 7.62$ (t, 7.4, 2H, H-14, H-16), 7.70 (t, 7.4, 1H, H-15), 7.80 (t, 7.6, $2 \mathrm{H}, \mathrm{H}-27, \mathrm{H}-29$ ), 7.89 (d, 8.5, 2H, H-21, H-23), 7.98 (dd, 8.2, $1.2,2 \mathrm{H}, \mathrm{H}-13, \mathrm{H}-17), 8.11$ (d, 8.5, 4H, H-8, H-10, H-20, H24), $8.30(\mathrm{~d}, 8.5,2 \mathrm{H}, \mathrm{H}-7, \mathrm{H}-11), 8.98(\mathrm{~s}, 1 \mathrm{H}, \mathrm{NH})$;

${ }^{13} \mathrm{C}-N M R$ (DMSO-d,$\delta \mathrm{ppm}$ ): 99.13 (C-22), 112.49 (C26, C-30), 120.45 (C-28), 127.58 (C-8, C-10), 127.65 (C-13, C-17), 128.54 (C-18), 129.86 (C-7, C-11), 129.33 (C-14, C16), 129.38 (C-27, C-29), 132.07 (C-6), 133.14 (C-19), 134.08 (C-15), 134.29 (C-20,C-24), 136.58 (C-5), 137.86 (C-21, C-23), 140.33 (C-12), 143.83 (C-9), 146.10 (C-25), 159.96 (C-3), 166.70 (C=0);

Anal. (\%): Calcd. for $\mathrm{C}_{28} \mathrm{H}_{20} \mathrm{IN}_{3} \mathrm{O}_{3} \mathrm{~S}(605.45 \mathrm{~g} / \mathrm{mol}): \mathrm{C}_{\text {, }}$ 55.55; H,3.33; N, 6.94; S, 5.30. Found: $C, 55.61 ; H, 3.27 \mathrm{~N}$, 6.90; S, 5.32;

APCl, m/z: $604[\mathrm{M}-\mathrm{H}]$;, $513\left[\mathrm{M}-\mathrm{H}-\mathrm{C}_{6} \mathrm{H}_{5} \mathrm{~N}\right] ;, 127$ ।

3-(4-(4-Clorophenylsulfonyl)phenyl)-5-(4-iodobenzylidene) -2-phenyl-1,2-dihydro-1,2,4-triazin-6(5H)-one $\mathbf{6 b}$

m.p. $=280-282{ }^{\circ} \mathrm{C}$; yield $=85 \%$

FT-IR $\left(\mathrm{KBr}, v \mathrm{~cm}^{-1}\right): 3281 \mathrm{~m}, 3087 \mathrm{w}, 3056 \mathrm{w}, 3040 \mathrm{~m}$, $1723 \mathrm{vs}, 1636 \mathrm{~s}, 1603 \mathrm{~m}, 1580 \mathrm{~s}, 1494 \mathrm{~m}, 1481 \mathrm{~m}, 1404 \mathrm{~m}$, $1327 \mathrm{~s}, 1310 \mathrm{~m}, 1291 \mathrm{~s}, 1154 \mathrm{vs}, 1103 \mathrm{~m}, 1088 \mathrm{~m}, 819 \mathrm{~m}, 760 \mathrm{~s}$, $575 \mathrm{~m}$

${ }^{1} \mathrm{H}-\mathrm{NMR}$ (DMSO-d ${ }_{6} \delta \mathrm{ppm}, \mathrm{J} \mathrm{Hz}$ ) : 6.73 (d, 7.8, 2H, H-26, $\mathrm{H}-30), 6.83$ (br t, 7.1, $1 \mathrm{H}, \mathrm{H}-28), 7.30(\mathrm{~s}, 1 \mathrm{H}, \mathrm{H}-18), 7.70$ (d, 8.5, 2H, H-14, H-16), 7.18 (t, 7.1, 2H, H-27, H-29), 7.89 (d, 8.3, 2H, H-21, H-23), 8.00 (d, 8.5, 2H, H-13, H-17), 8.12 (br d, 8.4, 4H, H-8, H-10, H-20, H-24), 8.30 (d, 8.4, 2H, H-7, H11), $8.99(\mathrm{~s}, 1 \mathrm{H}, \mathrm{NH})$;

${ }^{13} \mathrm{C}-\mathrm{NMR}$ (DMSO-d ${ }_{6} \delta \mathrm{ppm}$ ): 99.24 (C-22), 112.53 (C26, C-30), 120.50 (C-28), 128.67 (C-18), 127.79 (C-8, C10), 129.63 (C-13, C-17), 130.08 (C-7, C-11), 129.93 (C-14, C-16), 129.38 (C-27, C-29), 132.31 (C-6), 133.19 (C-19), 139.17 (C-15), 134.35 (C-20, C-24), 136.63 (C-5), 137.92 (C-21, C-23), 139.28 (C-12), 143.39 (C-9), 146.16 (C-25), 159.99 (C-3), $168.77(C=0)$;

Anal. (\%): Calcd. for $\mathrm{C}_{20} \mathrm{H}_{19} \mathrm{ClIN}_{3} \mathrm{O}_{3} \mathrm{~S}(639.89 \mathrm{~g} / \mathrm{mol}): \mathrm{C}$, 52.56; H, 2.99; N, 6.57; S, 5.01. Found: C, 52.64; H, 2.91; N, 6.62; S, 5.11;

APCl, m/z: $638\left[{ }^{35} \mathrm{Cl} \mathrm{M-H]} ; 640\left[{ }^{37} \mathrm{Cl} \mathrm{M-H]} ; 547[\mathrm{M}-\mathrm{H}-\right.\right.$ $\left.\mathrm{C}_{6} \mathrm{H}_{5} \mathrm{~N}\right] ; 549\left[\mathrm{M}-\mathrm{H}-\mathrm{C}_{6} \mathrm{H}_{5} \mathrm{~N}\right] ;, 127 \mathrm{I}$

3-(4-(4-Bromophenylsulfonyl) phenyl) -5-(4iodobenzylidene)-2-phenyl-1,2-dihydro-1,2,4-triazin-6(5H)one $6 \mathrm{c}$

m.p. $=281-282^{\circ} \mathrm{C} ;$ yield $=71 \%$ 
FT-IR $\left(\mathrm{KBr}, v \mathrm{~cm}^{-1}\right): 3282 \mathrm{~m}, 3087 \mathrm{w}, 3061 \mathrm{w}, 1722 \mathrm{~s}$, $1637 \mathrm{~s}, 1602 \mathrm{~m}, 1575 \mathrm{~s}, 1483 \mathrm{~s}, 1402 \mathrm{~m}, 1325 \mathrm{~s}, 1289 \mathrm{~s}$, $1156 \mathrm{vs}, 1102 \mathrm{~s}, 1068 \mathrm{~s}, 819 \mathrm{~m}, 615 \mathrm{~s}, 575 \mathrm{~m}$;

${ }^{1} \mathrm{H}-N M R\left(D M S O-\mathrm{d}_{6}, \delta \mathrm{ppm}, J \mathrm{~Hz}\right.$ ): 6.73 (br d, 7.9, 2H, H26, H-30), 6.82 (brt, 7.3, 1H, H-28), 7.29 (s, 1H, H-18), 7.84 (t, 8.6, 2H, H-14, H-16), 7.19 (brt t. 7.9, 2H, H-27, H-29), 7.90 (d, 8.2, 2H, H-21, H-23), 7.91 (d, 8.6, 2H, H-13, H-17), 8.11 (d, 8.2, 4H, H-8, H-10, H-20, H-24), 8.30 (d, 8.6, 2H, H-7, H11), $8.98(\mathrm{~s}, 1 \mathrm{H}, \mathrm{NH})$;

${ }^{13} \mathrm{C}-\mathrm{NMR}$ (DMSO-d,$\delta \mathrm{ppm}$ ): 99.15 (C-22), 112.47 (C26, C-30), 120.41 (C-28), 128.63 (C-18), 127.70 (C-8, C10), 129.29 (C-13, C-17), 129.35 (C-7, C-11), 132.99 (C-14, C-16), 129.61 (C-27, C-29), 132.30 (C-6), 133.17 (C-19), 128.40 (C-15), 134.37 (C-20, C-24), 136.62 (C-5), 137.89 (C-21, C-23), 139.58 (C-12), 143.34 (C-9), 146.22 (C-25), $159.98(\mathrm{C}-3), 168.75(\mathrm{C}=0)$;

Anal. (\%): Calcd. for $\mathrm{C}_{2} \mathrm{H}_{30} \mathrm{BrIN}_{3} \mathrm{O}_{3} \mathrm{~S}(684.34 \mathrm{~g} / \mathrm{mol}): \mathrm{C}$, 49.14; H, 2.80; N, 6.14; S, 4.69. Found: C, 49.30; H, 2.71; N, 6.03; S, 4.75;

APCl, m/z: 682 [ $\left.{ }^{79} \mathrm{Br} \mathrm{M}-\mathrm{H}\right] ; 684$ [ $\left.{ }^{81} \mathrm{Br} \mathrm{M}-\mathrm{H}\right] ;, 591[\mathrm{M}-\mathrm{H}-$ $\left.\mathrm{C}_{6} \mathrm{H}_{5} \mathrm{~N}\right] ;, 593\left[\mathrm{M}-\mathrm{H}-\mathrm{C}_{6} \mathrm{H}_{5} \mathrm{~N}\right] ;, 127 \mathrm{l}$

\section{Cytoxicity evaluation}

The cytotoxicity bioassay was performed for compounds $5 a-c, 6 a, b$ and intermediates $4 a-c$.

Both experiments were carried out in controlled environment at $25 \pm 1^{\circ} \mathrm{C}$, using a 16 -h photoperiod and $8 \mathrm{~h}$ of darkness using a climatic chamber (Sanyo MLR-351H; Sanyo, San Diego, CA, USA).

\section{Daphnia magna bioassay}

The bioassay was performed in duplicate according to the method described in our previous research [41]. Each determination was carried out in PP tissue culture wells (Greiner Bio-One) using 10 organisms in each well at a final volume of $4 \mathrm{~mL} / \mathrm{sample}$ and a final concentration of DMSO of $1 \%$, used as negative control. Seven compounds were tested at 6 concentration levels ranging from 8 to $198 \mathrm{ig} / \mathrm{mL}$. The lethality (L\%) was recorded over a $48 \mathrm{~h}$ period of exposure at two points: 24 and $48 \mathrm{~h}$. The $50 \%$ lethal concentrations (LC50) were computed for each compound by interpolation on lethality curves plotted between $L \%$ values and the logarithm of concentrations using the least square fit method. The LC50 and 95\% confidence intervals ( $\mathrm{Cl} 95 \%$ ) of $\mathrm{LC} 50$ were also calculated using the same method. All calculations were performed using the software GraphPad Prism v 5.1. The prediction of $\mathrm{LC} 5 \mathrm{O}_{48}$ was performed using the GUSAR software application.

\section{Artemia salina bioassay}

Brine shrimp lethality test was conducted according to the protocol described by Meyer et al. [36] with some modifications [42]. A. salina cysts were incubated in artificial sea water ( $40 \mathrm{~g} / \mathrm{L}$ salinity) for $24 \mathrm{~h}$ under continuous aeration. The new ly hatched nauplii were separated from the shells, transferred to fresh sea water and incubated for $24 \mathrm{~h}$. Each determination was carried out in PP tissue culture with 24-wells (Greiner Bio-One) using 10 organisms in each well at a final volume of $1 \mathrm{~mL} / \mathrm{sample}$ and a final concentration of DMSO of $1 \%$, used as negative control. The compounds were tested at the same levels of concentration as $D$. magna. The lethality $(\mathrm{L} \%)$ was recorded over a $24 \mathrm{~h}$ period of exposure and the calculations were made as presented above. No predictions could be performed for brine shrimps.

\section{Results and discussions \\ Chemistry}

The keyprecursor, 2-(4-(4-X-phenylsulfonyl) benzamido) acetic acids 4, previously reported in the literature [37,38], were obtained following the Steiger procedure consisting of N-acylation of glycine with 4-(4-X-phenylsulfonyl) benzoyl chlorides 3. The simultaneous condensation and cyclization reaction of $\mathrm{N}$-acylated glycine derivatives 4 with 4-iodobenzaldehyde, in acetic anhydride and in the presence of sodium acetate, led to the formation of new compounds from 4-iodobenzylidene-oxazole-5(4H)-ones 5. The 1,2,4-triazin- $6(5 H)$-ones 6 resulted through a ringopening and recyclization reaction of the oxazolones 5 with phenylhydrazine, in acetic acid and sodium acetate. The heterocyclic compounds were obtained in moderate yields, in a relatively short reaction time. The spectral data validated the structures of these compounds.

\section{IR Spectral data}

The reaction between $\mathrm{N}$-acylated glycine 4 and 4iodobenzaldehyde is firstly confirmed by the disappearance of the NH absorption band from the IR spectra of new compounds 5 that was present at $3416 \mathrm{~cm}^{-1}(4 \mathrm{a}), 3351$ $\mathrm{cm}^{-1}(\mathbf{4 b})$ and $3341 \mathrm{~cm}^{-1}(\mathbf{4 c})$ in precursors $4[37,38]$. The $\mathrm{C}=0$ lactone group from oxazolones 5 is highlighted in the IR spectra of these compounds by absorption bands due to stretching vibration, $v C=0$, at $1793-1796 \mathrm{~cm}^{-1}$ and 1769 $1772 \mathrm{~cm}^{-1}$ according to the literature for similar derivatives $[39,40]$.

The obtaining of triazinones from reaction of oxazolones with phenylhydrazine is firstly confirmed by appearance in the IR spectra of compounds 6 of a new absorption band at $3281 \mathrm{~cm}^{-1}$ in case of derivatives $6 a, b$ and at $3282 \mathrm{~cm}^{-1}$ in $6 \mathrm{c}$, characteristic to stretching vibration of $\mathrm{NH}$ group. The absorption band due to the stretching vibration of the $\mathrm{C}=0$ group appears at $1722 \mathrm{~cm}^{-1}$ for triazinones $6 \mathrm{a}, \mathrm{c}$ and at $1723 \mathrm{~cm}^{-1}$ for triazinone $6 \mathrm{~b}$.

\section{NMR Spectra data}

The ${ }^{1} \mathrm{H}-\mathrm{NMR}$ spectra of compounds 5 showed a singlet signal characteristic of $=\mathrm{CH}$ proton, with $\delta$ in the range

\begin{tabular}{|c|c|c|c|}
\hline $\begin{array}{c}\text { No. } \\
\text { compound }\end{array}$ & $\begin{array}{c}\text { Predicted LC50 } 48 \mathrm{~h} \\
(\mu \mathrm{g} / \mathrm{mL})\end{array}$ & $\begin{array}{l}\mathrm{LC50}_{45 \mathrm{~h}} \\
(\mu \mathrm{g} / \mathrm{mL})\end{array}$ & $\begin{array}{c}95 \% \text { CI of LC50 } 45 \mathrm{~h} \\
(\mu \mathrm{g} / \mathrm{mL})\end{array}$ \\
\hline $5 a$ & 0.27 & 56.15 & 35.37 to 89.13 \\
\hline $5 b$ & 0.17 & 355.5 & 179.3 to 704.7 \\
\hline $5 c$ & 0.16 & 111.7 & 93.49 to 133.4 \\
\hline $6 a$ & 0.19 & 178.2 & 138.7 to 229.0 \\
\hline $6 \mathrm{~b}$ & 0.08 & 159.1 & 135.2 to 187.1 \\
\hline $4 a$ & 28.96 & 219.1 & 142.1 to 337.9 \\
\hline $4 b$ & 15.27 & $\mathrm{NC}^{*}$ & $\mathrm{NC}^{*}$ \\
\hline $4 c$ & 4.01 & $\mathrm{NC}^{*}$ & $\mathrm{NC}^{*}$ \\
\hline
\end{tabular}

Table 1

LETALITY OF THE TESTED COMPOUNDS ON DAPHNIA MAGNA BIOASSAY 


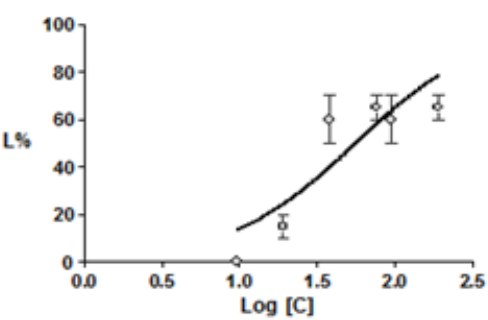

5 a

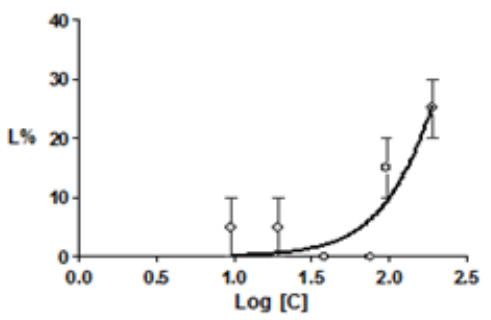

$5 b$

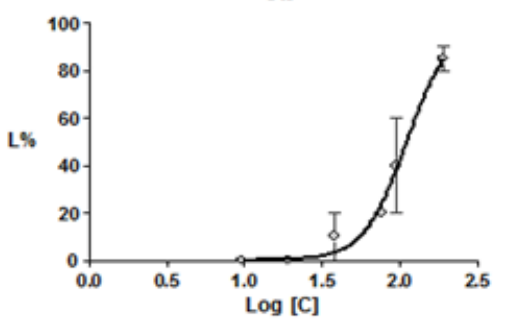

$5 \mathrm{c}$

7.39-7.41 ppm, which proves that the condensation reaction of $\mathrm{N}$-acylated glycine with 4-iodobenzaldehyde took place. Also, the absence of the triplet signal characteristic of the proton of $\mathrm{NH}$ group from compounds $4\left(\delta_{\mu H}=8.22-9.09 \mathrm{ppm}\right)[37,38]$ and of doublet signal of $\mathrm{CH}_{2}$ protons $\left(\delta_{\mathrm{CH}_{2}}=3.94-4.37 \mathrm{ppm}\right)$, represents another proof that the condensation reaction occurred simultaneously with the intramolecular cyclization.

In the ${ }^{1} \mathrm{H}-\mathrm{NMR}$ spectra of the compounds 6 , the presence of a new signal of NH proton from phenylhydrazine moiety, at $\sim 9.00 \mathrm{ppm}$, is the most important evidence that the reaction of oxazolone with phenylhydrazine occurred.

In the ${ }^{13} \mathrm{C}$-NMR spectra of the compounds 5 , the carbon atoms from oxazole ring were highlighted by the following signals that appeared atthe corresponding chemical shifts: 161.86-161.62 ppm for C-2, 132.92-133.47 ppm for the $>C=C H(C-4)$ and $166.25-166.46 \mathrm{ppm}$ for $\mathrm{C}=\mathrm{O}(\mathrm{C}-5)$, the last signal being the most deshielded, as expected.

In the ${ }^{13} \mathrm{C}$-NMR spectra of triazinones 6 , the $\mathrm{C}-3$ atom resonated at $\mathrm{a}=159.96-159.99 \mathrm{ppm}$, while the $>\mathrm{C}=\mathrm{CH}$ (C-5) at 136.58-136.63 ppm. The $C=0$ signal on the triazinone ring is observed in the range $166.70-168.77 \mathrm{ppm}$. Also, the methyne carbon from oxazolones 5 resonated at 131.22-132.62 ppm while the same carbon from the same position of triazinones 6 was appeared in region 128.54$128.67 \mathrm{ppm}$.

\section{Mass spectra}

Another support for the assigned structure of new compounds from oxazolones and triazinones classes was obtained by recording the mass spectra.

The mass spectra showed the presence of the molecular ions, thus confirming the structures of theese compounds. Fragmentation of oxazolones generally occurs by obtaining a carbonyl ion, stabilized by conjugation. For triazinones, the most characteristic fragmentation is the elimination of a neutral fragment with the formula $\mathrm{C}_{6} \mathrm{H}_{5} \mathrm{~N}$.
Fig. 3. Daphnia magna lethalithy curves at $48 \mathrm{~h}$ for tested compounds
For $\mathrm{Cl}$ and $\mathrm{Br}$ atoms, isotopic contributions were taken into account.

\section{Cytotoxicity evaluation}

Daphnia magna bioassay

The results are presented in table 1 and figure 3. At $24 \mathrm{~h}$, the lethality induced by the tested compounds was below $30 \%$ and LC50 couldn't be calculated. After $48 \mathrm{~h}$ of exposure, $\mathbf{4} \mathbf{b}$ and $\mathbf{4 c}$ did not produced aby lethal effects and no further calculations were possible. After calculating the LC50, the highest toxicity was exhibited by $5 \mathrm{a}$ and the lowest by $5 \mathbf{b}$. Among the tested compounds, 5 a had a LC50 lower than $100 \mu \mathrm{g} / \mathrm{mL}$, thus being a potent compound with biological activity on $D$. magna.

No correlation was found between GUSAR prediction values and experimental LC50 values. Moreover, 5b, 4b and $\mathbf{4 c}$ were found not to be toxic or exhibiting low toxicity, whereas the prediction software indicates them as potent cytotoxic compounds. By applying the t-student test on the two sets of data, predicted and experimentally obtained values, no similarity was found, $p$ value being $<0.0001$ which indicate a significant difference. A slightly correlation was found for compounds $\mathbf{4 a}$, and $\mathbf{4 b}$ regarding the order of magnitude.

\section{Artemia salina bioassay}

The toxicity at $24 \mathrm{~h}$ on $\mathrm{A}$. salina was lower than $25 \%$ for all compounds at the tested concentrations. The results are comparable to those obtained at $D$. magna bioasay. As showed previously [42], the brine shrimp lethality test is less sensitive than $D$. magna, mainly due to a better resistance of $A$. salina. Further tests will be performed to evaluate the toxicity of the compounds at $48 \mathrm{~h}$.

\section{Conclusions}

Six new heterocyclic compounds from oxazol-5(4H)ones and 1,2,4-triazin-6(5H)-ones classes containing a diarylsulfone moiety have been synthesized and 
characterized by spectral techniques and elemental analysis. The oxazolones have been obtained by the reaction of some 2-(4-(4-X-phenylsulfonyl) benzamido) acetic acids with 4-iodobenzaldehyde. The 1,2,4-triazin$6(5 H)$-ones were synthetized by treating 1,3-oxazol-5(4H)ones with phenylhydrazine.

The cytotoxicity bioassays on Daphnia magna and Artemia salina indicated that among the tested compounds oxazolone 5a showed moderate to high cytotoxicity on $D$. magna, thus being a good candidate for further biological activity screening, including anticancer and antimicrobial.

\section{References}

1. PALMER, D. C., Oxazoles: Synthesis, Reactions, and Spectroscopy, Part. B, J ohn Wiley \& Sons, Inc., New Jersey, USA, 2004, p. 208

2. EL-MEKABATY, A., Int. J. Modern Org. Chem., 2, no. 1, 2013, p. 40 3. JAT, L. R., MISHRA, R., PATHAK, D., Int. J. Pharm. Pharm. Sci., 4, no. 1, 2012, p. 378

4. ZHU, M., GOKHALE, V. M., SZABO, L., MUNOZ, R. M., BAEK, H., BASHYAM, S., HURLEY, L. H., Von HOFF, D. D., HAN, H., Mol. Cancer Ther., 6, no. 4, 2007, p. 1348

5. PARVEEN, M., ALI, A., AHMED, S., MALLA, A. M., ALAM, M., PEREIRA SILVA, P. S., RAMOS SILVA, M., LEE, D.-U., Spectrochim. Acta A, 104, 2013, p. 538

6. OLOMOLA, T. O., AKINBOYE, A. J., OLASUNKANMI, 0. O., OLASUNKANMI, L. O., Ife J ournal of Science, 20, no. 1, 2018, p. 1

7. AAGLAWE, M. J., DHULE, S. S., BAHEKAR, S. S., WAKTE, P. S., SHINDE, D. B., J. Korean Chem. Soc., 47, no. 2, 2003, p. 133

8. TAILE, V., HATZADE, K., GAIDHANE, P., INGLE, V., Turk. J. Chem., 33, 2009, p. 295

9. MULWAD, V. V., SATWE, D. S., Indian J. Chem., 2006, 45B, p. 1210

10. SAMAD, M. K., HAWAIZ, F. E., Bioorg. Chem., 85, 2019, p. 431

11. MOHAMED, L. W., EL-BADRY, O. M., EL-ANSARY, A. K., ISMAEL, A., Bioorg. Chem., 72, 2017, p. 308

12. KUS, C., UGURLU, E., OZDAMAR, E. D., CAN-EKE, B., Turk. J Pharm. Sci., 14, no. 2, 2017, p. 174

13. KHAN, K. M., MUGHAL, U. R., KHAN, M. T. H., ZIA-ULLAH, PERVEEN, S., CHOUDHARY, M. I., Bioorg. Med. Chem., 14, 2006, p. 6027 14. HAMIDIAN, H., TAGIZADEH, R., FOZOONI, S., ABBASALIPOUR, V., TAHERI, A., NAMJOU, M., Bioorg. Med. Chem., 21, 2013, p. 2088

15. ZAKI, I., ABDELHAMMEID, M. K., EL-DEEN, I. M., ABDEL WAHAB, A. H. A., ASHMAWY, A. M., MOHAMED, K. O., Eur. J. Med. Chem., 156, 2018, p. 563

16. EL-HADY, H. A., EL-SAKKA, S. S., Int. J. Innov. Res. Sci. Eng. Technol., 3, no. 4, 2014, p. 10854

17. ABU-MELHA, S., Spectrochim. Acta A, 96, 2012, p. 898

18. ABDEL-GALIL, E., MOAWAD, E. B., EL-MEKABATY, A., SAID, G. E., J. Heterocyclic Chem., 55, no. 5, 2018, p. 1092

19. SHAIKH, B. M., CHOBE, S. S., KONDA, S. G., KHANDARE, N. T., CHAVAN, S. A., DAWANE, B. S., Der Chemica Sinica, 1, no. 2, 2010, p. 86

20. KAUSHIK, D., KHAN, S. A., CHAWLA, G., Eur. J. Med. Chem. 45, 2010, p. 3960

21. BARBUCEANU, S.F., SARAMET, G., ALMAJAN, G. L., DRAGHICI, C., BARBUCEANU, F., BANCESCU, G., Eur. J. Med. Chem., 49, 2012, p. 417 22. BARBUCEANU, S. F., ILIES, D. C., SARAMET, G., UIVAROSI, V., DRAGHICl, C., RADULESCU, V., Int. J. Mol. Sci., 15, no. 6, 2014, p. 10908
23. BARBUCEANU, S. F., BANCESCU, G., CRETU, O. D., DRAGHICI, C., BANCESCU, A., RADU-POPESCU, M., Rev. Chim. (Bucharest), 61, no. 2, 2010, p. 140

24. APOSTOL, T. V., BARBUCEANU, S. F., OLARU, O. T., DRAGHICI, C., SARAMET, G., SOCEA, B., ENACHE, C., SOCEA, L.-I., Rev. Chim. (Bucharest), 70, no. 4, 2019, p. 1099

25. BARBUCEANU, S. F., BANCESCU, G., DRAGHICI, C., BARBUCEANU, F., CRETU, O. D., APOSTOL, T. V., BANCESCU, A., Rev. Chim. (Bucharest), 63, no. 4, 2012, p. 362

26. APOSTOL, T.-V., DRAGHICI, C., DINU, M., BARBUCEANU, S. F., SOCEA, L. I., SARAMET, I., Rev. Chim. (Bucharest), 62, no. 2, 2011, p. 142

27. BARBUCEANU, S. F., BANCESCU, G., SARAMET, G., BARBUCEANU, F., DRAGHICl, C., RADULESCU, F. S., IONESCU, A., NEGRES, S., Heteroat. Chem., 24, no. 4, 2013, p. 309

28. BARBUCEANU, S.-F., OLARU, O. T., SOCEA, L.-I., DRAGHICI, C., SARAMET, G., BARAITAREANU, S., SOCEA, B., BARBUCEANU, F., Rev. Chim. (Bucharest), 70, no. 1, 2019, p. 13

29. AHMAD, I., SHAGUFTA, Int. J. Pharm. Pharm. Sci., 7, no. 3, 2015, p. 19

30. AL-SAID M. S., GHORAB M. M., NISSAN Y. M., Chem. Cent. J., 6, 2012, p. 64

31. CHO, S. C., RHIM, J. H., SON, Y. H., LEE, S. J ., PARK, S. C., Exp. Mol. Med., 42, no. 3, 2010, p. 223.

32. GUILHERMINO, L., DIAMANTINO, T., SILVA, M. C., SOARES, A. M.V.M., Ecotoxicol. Environ. Saf., 46, 2000, p. 357

33. NITULESCU, G. M., PAUNESCU, H., DRAGHICI, C., MISSIR, A. V., COMAN, O. A., FULGA, I., Farmacia, 58, no. 2, 2010, p. 190

34. NITULESCU, G. M, IANCU G., NITULESCU, G., IANCU, R. C., BOGDANICI, C., VASILE, D., Rev. chim. (Bucharest), 68, no. 4, 2017, p. 754.

35. RADULESCU, C., STIHI, C., ILIE, M., LAZURCA, D., GRUIA, R., OLARU, O.T., BUTE, O.C., DULAMA, I.D., STIRBESCU, R.M., TEODORESCU, S., FLORESCU, M., Anal. Lett., 50, no. 17, 2017, p. 2839 36. MEYER, B. N., FERRIGNI, N. R., PUTNAM, J. E., J ACOBSEN, L. B., NICHOLS, D. E., MCLAUGHLIN, J. L., Planta Med., 45, no. 5, 1982, p. 31

37. SCHIKETANZ, I., DRAGHICl, C., SARAMET, I., BALABAN, A.T., Arkivoc, ii, 2002, p. 64

38. SCHIKETANZ, I., DRAGHICI, C., SARAMET, I., BALABAN, A. T., Rev. Roum. Chim., 47, no. 3-4, 2002, p. 235

39. RODRIGUES, C.A.B., MARIZ, I.F.A., MACOAS, E.M.S., AFONSO, C.A.M., MARTINHO, J.M.G., Dyes Pigments, 99, 2013, p. 642

40. SAOUR, K. Y., AL-BAYATI, R. I. H., SHIA, J. S., Chemistry and Materials Research, 7, no. 7, 2015, p. 105

41. NITULESCU, G., NICORESCU, I. M., OLARU, O. T., UNGURIANU, A., MIHAl, D. P., ZANFIRESCU, A., NITULESCU, G. M., MARGINA, D., Int. J. Mol. Sci., 18, no. 10, 2017, p. 2217

42. SEREMET, O. C., OLARU, O. T., GUTU, C. M., NITULESCU, G. M., ILIE, M., NEGRES, S., ZBARCEA, C. E., PURDEL, C. N., SPANDIDOS, D. A., TSATSAKIS, A. M., COLEMAN, M. D., MARGINA, D. M., Mol. Med. Rep., 17, no. 6, 2018, p. 7757

Manuscript received: 30.09 .2019 